\title{
THE STRIP DIELECTRIC BREAKDOWN MODEL
}

\author{
Tong-Yi Zhang*, Ming-Hao Zhao and Cun-Fa Gao \\ Department of Mechanical Engineering \\ Hong Kong University of Science and Technology \\ Clear Water Bay, Kowloon, Hong Kong, China
}

\begin{abstract}
This paper reports on the analysis of the strip dielectric breakdown (DB) model for an electrically impermeable crack in a piezoelectric medium based on the general linear constitutive equations. The DB model assumes that the electric field in a strip ahead of the crack tip is equal to the dielectric breakdown strength, which is in analogy with the classical Dugdale model for plastic yielding. Using the Stroh formalism and the dislocation modeling of a crack, we derived the relationship between the DB strip size and applied mechanical and electrical loads, the intensity factors of stresses and electric displacement, and the local energy release rate. Based on the results, we discussed the effect of electric fields on fracture of a transversely isotropic piezoelectric ceramic by applying the local energy release rate as a failure criterion. It is shown that for an impermeable crack perpendicular to the poling direction, a positive electric field will assist an applied mechanical stress to propagate the crack, while a negative electric field will retard crack propagation. However, for an impermeable crack parallel to the poling direction, it is found that the applied electric field does not change the mode I stress intensity factor and the local energy release rate, i.e., the applied electric field has no effect on the crack growth.
\end{abstract}

Keywords: Piezoelectric materials; Crack problem; Dielectric breakdown

\footnotetext{
*Corresponding author, Tel. +852-2358-7193, Fax +852-2358-1543, E-mail: mezhangt@ust.hk
} 


\section{Introduction}

In 1997, Gao et al. proposed a strip polarization saturation (PS) model by treating piezoelectric ceramics to be mechanically brittle and electrically ductile. The electric ductility is used to reflect the nonlinear relationship between the electric displacement and the electric field strength. The PS model takes the advantage that the constitutive relationship between the electric displacement and the electric field strength is similar to that between the stress and the strain. In the PS model, the electric displacement reaches a limited saturation value along a strip in front of an electrically impermeable crack. The PS strip completely and electrically shields the crack from applied mechanical and electrical loads such that the local intensity factor of electric displacement nulls. In the modeling Gao et al. (1997) employed a simplified constitutive equation, which made the analysis simple and the results easy to be understood. The corresponding analysis of the PS model based on the general linear constitutive equation was conducted and extended by Gao and Barnett (1996), Wang (2000), Ru (1999) and Zhang et al. (2002). McMeeking (2001) gave comprehensive and suggestive comments on the PS model. From the energy point of view, the electric displacement behaves like the strain, and the electric field strength like the mechanical strength. Therefore, the PS model does not correspond to the classical Dugdale model (Dugdal, 1960) in which the stress is equal to the yield strength along the strip in front of a crack tip. Instead, the PS model corresponds to a mechanical Dugdale model in which the strain remains a constant value as the stress increases. Very recently, Zhang and Gao (2004) proposed a strip dielectric breakdown (DB) model for an electrically impermeable crack of a semi-infinite length and Zhang (2004) further discussed the DB model with a finite length crack. The DB model is exactly analogous to the classical Dugdale model from the energy point of view because, in the DB model, the electric field strength on a strip adjacent to a crack tip is taken as a constant. The physical arguments for the DB model are described as follows. The theoretical results (Zhang and Tong, 1996; Zhang et al., 1998; Zhang et al. 2002) based on linear electro-elasticity show that the electric field is considerably high at 
the crack tip. Especially, if the electrically impermeable boundary conditions are approximately applied to an electrically insulated crack, the theoretical predicated electric field will approach infinity at the impermeable crack tip when applied electric field has a component in the direction perpendicular to the crack. Even when the electrically permeable boundary conditions are approximately applied to an electrically insulated crack, the theoretical predicated electric field, in terms of the electric field strength, will be about 1000 times higher in magnitude than the applied electric field for most piezoelectric materials because most piezoelectric materials have about a 1000 times higher dielectric constant in magnitude than the crack interior (air or vacuum). The local electric field may be much higher than the dielectric breakdown strength. The dielectric breakdown strength is defined as a critical electric field at which dielectric discharge occurs and leads to dielectric breakdown. The characteristics of the breakdown strength are similar to those of the mechanical fracture strength (Dissado and Fothergill, 1992), which is very sensitive to solid defects such as flaws, voids and cracks, thereby indicating that partial discharge may occur at the crack tip due to the high electric field. As a result, a local partial discharge zone or electric breakdown zone, like a plastic deformation zone, is formed adjacent to the crack tip, and in the partial discharge zone the electric field cannot exceed the dielectric breakdown strength, $E_{b}$, which is analogous to the yield strength in mechanically plastic deformation. In analogy with the mechanical Dugdale model, the dielectric breakdown region is assumed to be a strip along the crack's front line, where the electric field strength is equal to the dielectric breakdown strength, $E_{b}$. Therefore, the DB model proposed by Zhang and Gao (2004) and Zhang (2004) is exactly analogous to the classical Dugdale model (Dugdale, 1960) from the energy point of view.

In developing the DB model, however, Zhang and Gao (2004) and Zhang (2004) used the simplified electroelasticity constitutive equations to simplify analyses. Although such 
simplification makes it easy for one to capture a clear physical picture, it may lead to great error when the model is used to quantitative calculation of the energy release rate. In the present work, we study the DB model (Zhang and Gao, 2004; Zhang, 2004) based on the general linear constitutive equation. To compare the results from the generalized DB model with those of Gao et al. (1997), Zhang and Gao (2004), and Zhang (2004), a crack is still assumed to be electrically impermeable. Following the Stroh formalism in Section 2, we first outline the linear solutions of an impermeable crack, and then study the interaction of a piezoelectric dislocation with a finite impermeable crack in Sections 3 and 4, respectively, as prior works. In Section 5, we develop a generalized strip dielectric breakdown model by means of the dislocation modeling of a crack, and then present explicit expression for the local energy release rate. In Section 6, we use the local energy release rate as a failure criterion to discuss the effect of electric fields on fracture of piezoelectric ceramics. Finally, we conclude the present work in Section 7.

\section{Stroh formalism}

Following the previous overview articles (Zhang et al., 2002; Zhang and Gao, 2004), we briefly introduce the two-dimensional theoretical results for an electrically impermeable crack in a piezoelectric material based on linear electro-elasticity. Note that matrix and vector notations in this article are more consistent with those used in Zhang and Gao (2004), but may differ from the notations used in Zhang et al. (2002).

In a rectangular coordinate system, $x_{i}(i=1,2,3)$, the complete set of basic equations for a linear piezoelectric solid without any body forces and free charges is given by the equilibrium equation, Eq. (1), and the kinematic equation, Eq. (2), and the constitutive equation, Eq. (3) (Zhang et al., 2002; Zhang and Gao, 2004): 


$$
\begin{aligned}
& \sigma_{i j, j}=0, \quad D_{i, i}=0, \\
& \varepsilon_{i j}=\frac{1}{2}\left(u_{i, j}+u_{j, i}\right), \quad E_{i}=-\varphi_{, i}, \\
& \sigma_{i j}=C_{i j k l} \varepsilon_{k l}-e_{k i j} E_{k}, \\
& D_{k}=e_{k i j} \varepsilon_{i j}+\kappa_{k l} E_{l},
\end{aligned}
$$

where $\sigma_{i j}, \varepsilon_{i j}, D_{i}$ and $E_{i}$ denote stress tensor, strain tensor, electric displacement vector and electric field vector, respectively; $u_{i}$ and $\varphi$ are the elastic displacements and electric potential, respectively; and $C_{i j k l}, e_{i j k}$ and $\kappa_{i j}$ stand for the elastic constants, the piezoelectric constants and the dielectric constants, respectively.

For generalized two-dimensional deformations in which the generalized displacement vector $\mathbf{u}=\left(\begin{array}{llll}u_{1} & u_{2} & u_{3} & \varphi\end{array}\right)^{T}$ depends on $x_{1}$ and $x_{2}$ only, the general solution takes the form (Zhang et al., 2002; Zhang and Gao, 2004):

$$
\begin{aligned}
& \mathbf{u}=\mathbf{A f}(z)+\overline{\mathbf{A}} \overline{\mathbf{f}(z)}, \\
& \phi=\mathbf{B f}(z)+\overline{\mathbf{B}} \overline{\mathbf{f}(z)},
\end{aligned}
$$

where $\mathbf{A}=\left(\begin{array}{llll}\mathbf{a}_{1} & \mathbf{a}_{2} & \mathbf{a}_{3} & \mathbf{a}_{4}\end{array}\right)$ and $\mathbf{B}=\left(\begin{array}{llll}\mathbf{b}_{1} & \mathbf{b}_{2} & \mathbf{b}_{3} & \mathbf{b}_{4}\end{array}\right)$ with $\mathbf{a}_{\alpha}$ and $\mathbf{b}_{\alpha}$ for $\alpha=1,2,3,4$ being both four dimensional eigen-vectors, $\mathbf{f}(\mathrm{z})=\left(\begin{array}{lllll}f_{1}\left(z_{1}\right) & f_{2}\left(z_{2}\right) & f_{3}\left(z_{3}\right) & f_{4}\left(z_{4}\right)\end{array}\right)^{T}$ is an analytic function vector, $z_{\alpha}=x_{1}+p_{\alpha} x_{2}$, and $p_{\alpha}$ is a complex eigen-root with a positive imaginary part, and $\phi$ is the generalized stress function vector such that

$$
\begin{aligned}
& \Sigma_{2}=\left(\begin{array}{llll}
\sigma_{21} & \sigma_{22} & \sigma_{23} & D_{2}
\end{array}\right)^{T}=\phi_{, 1}, \\
& \Sigma_{1}=\left(\begin{array}{llll}
\sigma_{11} & \sigma_{12} & \sigma_{13} & D_{1}
\end{array}\right)^{T}=-\phi_{, 2} .
\end{aligned}
$$

It is convenient to calculate $p_{\alpha}$ by solving the following standard eigen-equation: 


$$
\left(\begin{array}{ll}
\mathbf{N}_{1} & \mathbf{N}_{2} \\
\mathbf{N}_{3} & \mathbf{N}_{1}^{T}
\end{array}\right)\left(\begin{array}{l}
\mathbf{a} \\
\mathbf{b}
\end{array}\right)=p\left(\begin{array}{l}
\mathbf{a} \\
\mathbf{b}
\end{array}\right)
$$

where $\mathbf{N}_{1}=-\mathbf{T}^{-1} \mathbf{R}^{T}, \mathbf{N}_{2}=\mathbf{T}^{-1}=\mathbf{N}_{2}^{T}, \mathbf{N}_{3}=\mathbf{R} \mathbf{T}^{-1} \mathbf{R}^{T}-\mathbf{Q}=\mathbf{N}_{3}^{T}$, and $\mathbf{Q}=\left(\begin{array}{cc}C_{i 1 k 1} & e_{11 i} \\ e_{11 i}^{T} & -\kappa_{11}\end{array}\right)$, $\mathbf{R}=\left(\begin{array}{cc}C_{i 1 k 2} & e_{21 i} \\ e_{12 i}^{T} & -\kappa_{12}\end{array}\right), \mathbf{T}=\left(\begin{array}{cc}C_{i 2 k 2} & e_{22 i} \\ e_{22 i}^{T} & -\kappa_{22}\end{array}\right), i, k=1,2,3$. The $\mathbf{A}$ and $\mathbf{B}$ matrices have the following relationship (Zhang et al., 2002; Zhang and Gao, 2004):

$$
\left(\begin{array}{ll}
\mathbf{B}^{T} & \mathbf{A}^{T} \\
\overline{\mathbf{B}}^{T} & \overline{\mathbf{A}}^{T}
\end{array}\right)\left(\begin{array}{ll}
\mathbf{A} & \overline{\mathbf{A}} \\
\mathbf{B} & \overline{\mathbf{B}}
\end{array}\right)=\left(\begin{array}{ll}
\mathbf{I} & 0 \\
0 & \mathbf{I}
\end{array}\right)
$$

where $\mathbf{I}$ is a $4 \times 4$ unit matrix. In addition, two matrices, $\mathbf{Y}$ and $\mathbf{H}$, are often used in the following analysis, which are defined by

$$
\begin{gathered}
\mathbf{Y}=i \mathbf{A B}^{-1}, \\
\mathbf{H}=2 \operatorname{Re}[\mathbf{Y}] .
\end{gathered}
$$

Matrix $\mathbf{Y}$ is a Hermitian matrix and can be partitioned into

$$
\mathbf{Y}=\left(\begin{array}{cc}
\mathbf{Y}_{e} & \mathbf{Y}_{31} \\
\mathbf{Y}_{13} & Y_{44}
\end{array}\right)
$$

where the upper left block, $\mathbf{Y}_{e}$, is a $3 \times 3$ matrix, and $Y_{44}$ is a real element. For a stable material, $\mathbf{Y}_{e}$ is positive definite and $Y_{44}<0$, which leads to $H_{44}<0$.

\section{Solutions for a finite impermeable crack under applied loading}

Consider an electrically impermeable crack, as shown in Fig.1, lying on the $x_{1}$-axis from $-a$ to $a$ in a piezoelectric material under remotely uniform electrical and/or mechanical loading. The mechanical traction-free and the electrically impermeable boundary conditions require

$$
\Sigma_{2}=\left(\begin{array}{llll}
\sigma_{21} & \sigma_{22} & \sigma_{23} & D_{2}
\end{array}\right)^{T}=\phi_{, 1}=0, \quad \text { for }-a<x_{1}<a .
$$


The analytic function, $\mathbf{f}(z)$, satisfying the boundary conditions along the crack faces is give by (Zhang et al., 2002)

$$
\begin{aligned}
& f_{\alpha}=\frac{\left(a_{1}\right)_{\alpha}\left(z_{\alpha}+\sqrt{z_{\alpha}^{2}-a^{2}}\right)}{2}+\frac{a^{2}\left(a_{2}\right)_{\alpha}}{2\left(z_{\alpha}+\sqrt{z_{\alpha}^{2}-a^{2}}\right)}, \\
& f_{\alpha, 1}=\left(\left(a_{1}\right)_{\alpha}-\frac{a^{2}\left(a_{2}\right)_{\alpha}}{\left(z_{\alpha}+\sqrt{z_{\alpha}^{2}-a^{2}}\right)^{2}}\right) \frac{z_{\alpha}+\sqrt{z_{\alpha}^{2}-a^{2}}}{2 \sqrt{z_{\alpha}^{2}-a^{2}}}, \\
& f_{\alpha, 2}=p_{\alpha} f_{\alpha, 1}, \quad \alpha=1,2,3,4(\alpha \text { not summed }),
\end{aligned}
$$

where $\mathbf{a}_{1}$ and $\mathbf{a}_{2}$ are both four dimensional vectors with the relationship of $\mathbf{B} \mathbf{a}_{2}+\overline{\mathbf{B a}} \mathbf{a}_{1}=0$, and $\mathbf{a}_{1}$ is determined by the remote loading conditions,

$$
\begin{aligned}
& \left(\begin{array}{llll}
\sigma_{12}^{\infty} & \sigma_{22}^{\infty} & \sigma_{32}^{\infty} & D_{2}^{\infty}
\end{array}\right)^{T}=\mathbf{B} \mathbf{a}_{1}+\overline{\mathbf{B} \mathbf{a}_{1}}, \\
& \left(\begin{array}{cccc}
\sigma_{11}^{\infty} & \sigma_{21}^{\infty} & \sigma_{31}^{\infty} & D_{1}^{\infty}
\end{array}\right)^{T}=-\mathbf{B}\left\langle p_{\alpha}\right\rangle \mathbf{a}_{1}-\overline{\mathbf{B}\left\langle p_{\alpha}\right\rangle \mathbf{a}_{1}} .
\end{aligned}
$$

Because there are only seven independent remote input loads in Eq. (13), we take the null rotation around the $x_{3}$-axis at infinity as a supplementary condition (Zhang et al., 2002) by requiring

$$
\mathbf{A}_{2} \mathbf{a}_{1}-\left(\mathbf{A}\left\langle p_{\alpha}\right\rangle\right)_{1} \mathbf{a}_{1}+\overline{\mathbf{A}_{2} \mathbf{a}_{1}}-\overline{\left(\mathbf{A}\left\langle p_{\alpha}\right\rangle\right)_{1} \mathbf{a}_{1}}=0
$$

Note that a constant vector, $\mathbf{f}_{0}$, can be added into the solution of $\mathbf{f}(z)$ in Eq. (12). The constant vector, $\mathbf{f}_{0}$, is related to the reference states of the generalized displacements and the generalized stress functions, but does not affect the stress and electric fields. Therefore, we may ignore the constant vector, $\mathbf{f}_{0}$, and its associated constant generalized displacement and stress function vectors for simplicity.

We define an intensity factor vector at the right crack tip as

$$
\mathbf{K}^{*}=\lim _{z_{\alpha} \rightarrow a} \mathbf{B}\left\langle\sqrt{2 \pi\left(z_{\alpha}-a\right)}\right\rangle \mathbf{f}_{, 1} .
$$

Equation (15) gives 


$$
\mathbf{f}_{, 1}=\left\langle\frac{1}{\sqrt{2 \pi z_{\alpha}^{*}}}\right\rangle \mathbf{B}^{-1} \mathbf{K}^{*} \quad \text { for }\left|z_{\alpha}^{*}\right|<<a
$$

where $z_{\alpha}^{*}=z_{\alpha}-a$ and $\left|z_{\alpha}^{*}\right|$ is the absolute value of the complex variable $z_{\alpha}^{*}$. For $\left|z_{\alpha}^{*}\right|<<a$, we consequently have

$$
\begin{aligned}
& \mathbf{f}=\left\langle\frac{2 \sqrt{z_{\alpha}^{*}}}{\sqrt{2 \pi}}\right\rangle \mathbf{B}^{-1} \mathbf{K}^{*}, \\
& \mathbf{f}_{, 2}=\left\langle\frac{p_{\alpha}}{\sqrt{2 \pi z_{\alpha}^{*}}}\right\rangle \mathbf{B}^{-1} \mathbf{K}^{*} .
\end{aligned}
$$

A substitution of Eq. (12) into Eq. (15) yields

$$
\mathbf{K}^{*}=\frac{\sqrt{\pi a}}{2}\left(\begin{array}{c}
\sigma_{12}^{\infty} \\
\sigma_{22}^{\infty} \\
\sigma_{32}^{\infty} \\
D_{2}^{\infty}
\end{array}\right) \equiv \frac{1}{2}\left(\begin{array}{c}
K_{I I} \\
K_{I} \\
K_{I I I} \\
K_{D}
\end{array}\right)=\frac{1}{2} \mathbf{K},
$$

which shows that the intensity factor vector $\mathbf{K}^{*}$ is real and its components equal half of the stress intensity factors of mode II, I, and III and the electric displacement intensity factor. Then, the mechanical and electrical fields near the crack tip can be approximately expressed by

$$
\begin{aligned}
& \mathbf{u}=\frac{1}{\sqrt{2 \pi}}\left[\mathbf{A}\left\langle\sqrt{z_{\alpha}^{*}}\right\rangle \mathbf{B}^{-1}+\overline{\mathbf{A}}\left\langle\sqrt{\overline{z_{\alpha}^{*}}}\right) \overline{\mathbf{B}^{-1}}\right] \mathbf{K}, \\
& \Sigma_{2}=\frac{1}{2 \sqrt{2 \pi}}\left[\mathbf{B}\left(\frac{1}{\sqrt{z_{\alpha}^{*}}}\right) \mathbf{B}^{-1}+\overline{\mathbf{B}}\left(\frac{1}{\sqrt{\frac{z_{\alpha}^{*}}{}}}\right) \overline{\mathbf{B}^{-1}}\right] \mathbf{K}, \\
& \Sigma_{1}=-\frac{1}{2 \sqrt{2 \pi}}\left[\mathbf{B}\left(\frac{p_{\alpha}}{\sqrt{z_{\alpha}^{*}}}\right) \mathbf{B}^{-1}+\overline{\mathbf{B}}\left(\frac{\overline{p_{\alpha}}}{\sqrt{\frac{z_{\alpha}^{*}}{k}}}\right) \overline{\mathbf{B}^{-1}}\right] \mathbf{K} .
\end{aligned}
$$

The generalized crack opening near the crack tip is

$$
\Delta \mathbf{u}=\frac{\sqrt{2 r}}{\sqrt{\pi}} \mathbf{H K}
$$


Using the J-integral (Zhang et al., 2002; Cherepavon, 1979)

$$
J=\int_{\Gamma}\left(h n_{1}-\sigma_{i j} n_{j} u_{i, 1}+D_{i} E_{1} n_{i}\right) d \Gamma,
$$

where

$$
h=\frac{1}{2} \sigma_{i j} \varepsilon_{i j}-\frac{1}{2} D_{i} E_{i}
$$

denotes the electric enthalpy per unit volume, we obtain the J-integral or the energy release rate

$$
G=J=\mathbf{K}^{T} \frac{\mathbf{H}}{4} \mathbf{K}
$$

\section{Interaction of a piezoelectric dislocation with a finite impermeable crack}

For a piezoelectric dislocation located at $z_{\alpha}^{d}$ in an infinite piezoelectric medium, the analytic function vector is given by

$$
\mathbf{f}(z)=\left\langle\ln \left(z_{\alpha}-z_{\alpha}^{d}\right)\right\rangle \mathbf{q},
$$

where the $\mathbf{q}$ vector represents the feature of a generalized piezoelectric dislocation and combines the generalized Burgers vector, $\mathbf{b}$, and the generalized force, $\mathbf{F}$,

$$
\mathbf{q}=\frac{1}{2 \pi i}\left(\mathbf{A}^{T} \mathbf{F}+\mathbf{B}^{T} \mathbf{b}\right)
$$

The generalized Burgers vector, $\mathbf{b}$, and the generalized force, $\mathbf{F}$, are defined as $\mathbf{b}=\left(\begin{array}{llll}b_{1} & b_{2} & b_{3} & \Delta \varphi\end{array}\right)^{T}$ and $\mathbf{F}=\left(\begin{array}{llll}F_{1} & F_{2} & F_{3} & -q\end{array}\right)^{T}$, respectively, where $b_{i}$ and $F_{i}$ denote the component of the Burgers vector and a line force per unit length along the $x_{i}$ direction, respectively, $\Delta \varphi$ is the electric potential jump, and $q$ stands for a line charge per unit length. 
When the dislocation is near a finite length impermeable crack, the solution, satisfying the boundary conditions of Eq. (11) along the impermeable crack faces, takes the form:

$$
\begin{aligned}
& \mathbf{f}_{, 1}=\left\langle\frac{1}{\sqrt{z_{\alpha}^{2}-a^{2}}}\left(\frac{z_{\alpha}+\sqrt{z_{\alpha}^{2}-a^{2}}}{z_{\alpha}+\sqrt{z_{\alpha}^{2}-a^{2}}-z_{\alpha}^{d}-\sqrt{\left(z_{\alpha}^{d}\right)^{2}-a^{2}}}\right)\right\rangle \mathbf{q}+\mathbf{Z}_{, 1} \overline{\mathbf{q}}, \\
& Z_{i j, 1}=\sum_{k=1}^{4} B_{i k}^{-1} \frac{1}{B_{k j}} \frac{1}{\sqrt{z_{i}^{2}-a^{2}}}\left(\frac{a^{2}}{a^{2}-\left(z_{i}+\sqrt{z_{i}^{2}-a^{2}}\right)\left(\overline{z_{j}^{d}}+\sqrt{\left(\overline{z_{j}^{d}}\right)^{2}-a^{2}}\right)}\right), \\
& \mathbf{f}_{, 2}=\left\langle\frac{p_{\alpha}}{\sqrt{z_{\alpha}^{2}-a^{2}}}\left(\frac{z_{\alpha}+\sqrt{z_{\alpha}^{2}-a^{2}}}{z_{\alpha}+\sqrt{z_{\alpha}^{2}-a^{2}}-z_{\alpha}^{d}-\sqrt{\left(z_{\alpha}^{d}\right)^{2}-a^{2}}}\right)\right\rangle \mathbf{q}+\mathbf{Z}_{, 2} \overline{\mathbf{q}}, \\
& Z_{i j, 2}=\sum_{k=1}^{4} B_{i k}^{-1} \overline{B_{k j}} \frac{p_{i}}{\sqrt{z_{i}^{2}-a^{2}}}\left(\frac{a^{2}}{a^{2}-\left(z_{i}+\sqrt{z_{i}^{2}-a^{2}}\right)\left(\overline{z_{j}^{d}}+\sqrt{\left(\overline{z_{j}^{d}}\right)^{2}-a^{2}}\right)}\right) .
\end{aligned}
$$

The intensity factor vector at the right crack tip induced by the dislocation at $z_{\alpha}^{d}$ is

$$
\begin{aligned}
& \mathbf{K}^{*}=\lim _{z_{\alpha} \rightarrow a} \mathbf{B}\left\langle\sqrt{2 \pi\left(z_{\alpha}-a\right)}\right\rangle \mathbf{f}_{, 1} \\
& =-\sqrt{\pi a}\left[\mathbf{B}\left\langle\frac{1}{z_{\alpha}^{d}-a+\sqrt{\left(z_{\alpha}^{d}\right)^{2}-a^{2}}}\right\rangle \mathbf{q}+\overline{\mathbf{B}}\left\langle\frac{1}{\overline{z_{\alpha}^{d}}-a+\sqrt{\left(\overline{z_{\alpha}^{d}}\right)^{2}-a^{2}}}\right) \overline{\mathbf{q}}\right] .
\end{aligned}
$$

Equation (26) indicates that the intensity factors induced by a dislocation are real such that $\mathbf{K}^{*}=\mathbf{K} / 2$ holds. As a consequence, Eqs. (19-22) give also the crack tip fields and the energy release rate produced by the piezoelectric dislocation, respectively. 


\section{Dielecrtic breakdown model}

Following the successful treatment of the problem of plastic yielding at a crack tip by Dugdale (1960), Bilby et al. (1963) developed a dislocation model to formulate the strip plastic yielding. In the dislocation model (Bilby et al. 1963), the crack and the strip plastic zone are simulated by an array of dislocations. We shall adopt the dislocation approach to develop the DB model.

First, we consider a conventional dislocation, which has only the Burgers vector, $\mathbf{b}^{*}=\left(\begin{array}{lll}b_{1} & b_{2} & b_{2}\end{array}\right)^{T}$, and a pure electric dislocation, which is characterized by a nonzero value of $\Delta \varphi$. Hereafter, we shall use the subscripts, " $m$ " and " $e$ ", to denote properties associated with the conventional dislocation and the electric dislocation, respectively. For example,

$$
\begin{aligned}
& \mathbf{q}_{m}=\frac{1}{2 \pi i} \mathbf{B}^{T}\left(\begin{array}{c}
\mathbf{b}^{*} \\
0
\end{array}\right), \\
& \mathbf{q}_{e}=\frac{1}{2 \pi i} \mathbf{B}^{T}\left(\begin{array}{c}
0 \\
\Delta \varphi
\end{array}\right),
\end{aligned}
$$

If a conventional dislocation is located at $x_{1}^{d}$ on the $x_{1}$-axis in an infinite body without any crack, the dislocation induces a generalized stress, $\boldsymbol{\Sigma}_{2, m}$, and an electric strength component, $E_{2, m}$, along the $x_{1}$-axis:

$$
\begin{aligned}
& \boldsymbol{\Sigma}_{2, m}=\frac{1}{x_{1}-x_{1}^{d}} \frac{1}{2 \pi i}\left(\mathbf{B} \mathbf{B}^{T}-\overline{\mathbf{B}} \overline{\mathbf{B}}^{T}\right)\left(\begin{array}{c}
\mathbf{b}^{*} \\
0
\end{array}\right) \equiv \frac{1}{\pi\left(x_{1}-x_{1}^{d}\right)}\left(\begin{array}{l}
\mathbf{F}_{1} \\
\mathbf{F}_{2}
\end{array}\right) \mathbf{b}^{*}, \\
& E_{2, m}=-\frac{1}{x_{1}-x_{1}^{d}} \frac{1}{2 \pi i}\left(\mathbf{A}\left\langle p_{\alpha}\right\rangle \mathbf{B}^{T}-\overline{\mathbf{A}}\left\langle\bar{p}_{\alpha}\right\rangle \overline{\mathbf{B}}^{T}\right)_{4}\left(\begin{array}{c}
\mathbf{b}^{*} \\
0
\end{array}\right) \equiv \frac{1}{\pi\left(x_{1}-x_{1}^{d}\right)} \mathbf{G}_{1} \mathbf{b}^{*},
\end{aligned}
$$


where $\left(\mathbf{F}_{1}\right)_{k l}=\frac{1}{2 i}\left(\mathbf{B} \mathbf{B}^{T}-\overline{\mathbf{B}}^{T}\right)_{k l}$ for $k, l=1,2,3$ is a $3 \times 3$ matrix, $\left(\mathbf{F}_{2}\right)_{l}=\frac{1}{2 i}\left(\mathbf{B} \mathbf{B}^{T}-\overline{\mathbf{B}}^{T} \overline{\mathbf{B}}_{4 l}\right.$ and $\left(\mathbf{G}_{1}\right)_{l}=\frac{1}{2 i}\left(\mathbf{A}\left\langle p_{\alpha}\right\rangle \mathbf{B}^{T}-\overline{\mathbf{A}}\left\langle\bar{p}_{\alpha}\right\rangle \overline{\mathbf{B}}^{T}\right)_{4 l}$ for $l=1,2,3$ are two row vectors with each having three elements. Similarly, if an electric dislocation is located at $x_{1}^{d}$ on the $x_{1}$-axis in an infinite body without any crack, the dislocation induces a generalized stress, $\boldsymbol{\Sigma}_{2, e}$, and an electric strength component, $E_{2, e}$, along the $x_{1}$-axis:

$$
\begin{gathered}
\boldsymbol{\Sigma}_{2, e}=\frac{1}{x_{1}-x_{1}^{d}} \frac{1}{2 \pi i}\left(\mathbf{B B}^{T}-\overline{\mathbf{B}} \overline{\mathbf{B}}^{T}\right)\left(\begin{array}{c}
0 \\
\Delta \varphi
\end{array}\right) \equiv \frac{1}{\pi\left(x_{1}-x_{1}^{d}\right)}\left(\begin{array}{c}
\mathbf{F}_{2}^{T} \\
F_{44}
\end{array}\right) \Delta \varphi, \\
E_{2, e}=-\frac{1}{x_{1}-x_{1}^{d}} \frac{1}{2 \pi i}\left(\mathbf{A}\left\langle p_{\alpha}\right\rangle \mathbf{B}^{T}-\overline{\mathbf{A}}\left\langle\bar{p}_{\alpha}\right\rangle \overline{\mathbf{B}}^{T}\right)_{4}\left(\begin{array}{c}
0 \\
\Delta \varphi
\end{array}\right) \equiv \frac{1}{\pi\left(x_{1}-x_{1}^{d}\right)} G_{2} \Delta \varphi,
\end{gathered}
$$

where $F_{44}=\frac{1}{2 i}\left(\mathbf{B} \mathbf{B}^{T}-\overline{\mathbf{B}} \overline{\mathbf{B}}^{T}\right)_{44}$ and $G_{2}=\frac{1}{2 i}\left(\mathbf{A}\left\langle p_{\alpha}\right\rangle \mathbf{B}^{T}-\overline{\mathbf{A}}\left\langle\bar{p}_{\alpha}\right\rangle \overline{\mathbf{B}}^{T}\right)_{44}$ are two scalars. From Eqs. (8) and (9), we have

such that

$$
\frac{1}{2 i}\left(\mathbf{B B}^{T}-\overline{\mathbf{B}} \overline{\mathbf{B}}^{T}\right)=\mathbf{H}^{-1}
$$

$$
\mathbf{H}^{-1}=\left(\begin{array}{ll}
\mathbf{F}_{1} & \mathbf{F}_{2}^{T} \\
\mathbf{F}_{2} & F_{44}
\end{array}\right) .
$$

Eqs. (28) and (29) are the Green functions for the formulations of the DB model.

Figure 2 shows the DB model (Zhang and Gao, 2004; Zhang, 2004), where $(-c, a)$ and $(a, c)$ denote the dielectric breakdown strips. The external loading is applied uniformly at infinity. The boundary conditions along the crack faces are the same as these given by Eq. (11), while the boundary conditions along the dielectric breakdown strips are

$$
u_{i}\left(x_{1}, 0^{+}\right)=u_{i}\left(x_{1}, 0^{-}\right), \quad E_{2}\left(x_{1}, 0^{+}\right)=E_{2}\left(x_{1}, 0^{-}\right)=E_{b}, \quad a<\left|x_{1}\right|<c .
$$

where $E_{b}$ is the breakdown electric strength. 
We introduce four distribution functions, $g_{i}\left(x_{1}\right)$ for $i=1,2,3,4$, that are correspondingly associated with the Burgers vector components, $b_{1}, b_{2}, b_{3}$, and the jump of electric potential, $\Delta \varphi$, such that $g_{i}\left(x_{1}\right) b_{i} d x_{1}$, for $i=1,2,3,4$ and $b_{4} \equiv \Delta \varphi$, represents the strength of the Burgers vector located at $x_{1}$ in the interval $d x_{1}$. Thus, using the Green functions and the boundary conditions of Eqs. (11) and (32), we have the following integral equations

$$
\begin{aligned}
& \int_{-a}^{a} \frac{1}{\pi\left(x_{1}-x_{1}^{\prime}\right)} \mathbf{F}_{1}\left\langle g_{i}\right\rangle \mathbf{b}^{*} d x_{1}^{\prime}+\int_{-c}^{c} \frac{1}{\pi\left(x_{1}-x_{1}^{\prime}\right)} \mathbf{F}_{2}^{T} g_{4} \Delta \varphi d x_{1}^{\prime}+\mathbf{t}^{*}=0, \quad\left|x_{1}\right| \leq a, \\
& \int_{-a}^{a} \frac{1}{\pi\left(x_{1}-x_{1}^{\prime}\right)} \mathbf{F}_{2}\left\langle g_{i}\right\rangle \mathbf{b}^{*} d x_{1}^{\prime}+\int_{-c}^{c} \frac{1}{\pi\left(x_{1}-x_{1}^{\prime}\right)} F_{44} g_{4} \Delta \varphi d x_{1}^{\prime}+D_{2}^{\infty}=0, \quad\left|x_{1}\right| \leq a, \\
& \int_{-a}^{a} \frac{1}{\pi\left(x_{1}-x_{1}^{\prime}\right)} \mathbf{G}_{1}\left\langle g_{i}\right\rangle \mathbf{b}^{*} d x_{1}^{\prime}+\int_{-c}^{c} \frac{1}{\pi\left(x_{1}-x_{1}^{\prime}\right)} G_{2} g_{4} \Delta \varphi d x_{1}^{\prime}+E_{2}^{\infty}=E_{b}, \quad a \leq\left|x_{1}\right| \leq c,
\end{aligned}
$$

where $\left\langle g_{i}\left(x_{1}\right)\right\rangle, i=1,2,3$, is a $3 \times 3$ diagonal matrix and

$$
\mathbf{t}=\left(\begin{array}{cccc}
\sigma_{12}^{\infty} & \sigma_{22}^{\infty} & \sigma_{32}^{\infty} & D_{2}^{\infty}
\end{array}\right)^{T}=\left(\begin{array}{ll}
\mathbf{t}^{* T} & D_{2}^{\infty}
\end{array}\right)^{T}, \quad \mathbf{t}^{*}=\left(\begin{array}{ccc}
\sigma_{12}^{\infty} & \sigma_{22}^{\infty} & \sigma_{32}^{\infty}
\end{array}\right)^{T}
$$

In Eq. (33) the Cauchy principal values of the integral are to be taken at $x_{1}=x_{1}^{\prime}$ to avoid divergence and the same treatment should be taken in following integral equations. The conventional dislocations are distributed along the crack, from $-a$ to $a$, whereas the range of the electric dislocation distribution is from $-c$ to $c$, covering the DB zones and the crack. Substituting Eq. (33b) into (33a) yields

$$
\int_{-a}^{a} \frac{1}{\pi\left(x_{1}-x_{1}^{\prime}\right)} \mathbf{F}_{1}^{*}\left\langle g_{i}\right\rangle \mathbf{b}^{*} d x_{1}^{\prime}+\mathbf{T}^{*}=0, \quad\left|x_{1}\right| \leq a,
$$

where

$$
\mathbf{F}_{1}^{*}=\mathbf{F}_{1}-\mathbf{F}_{2}^{T} \mathbf{F}_{2} / F_{44}, \quad \mathbf{T}^{*}=\mathbf{t}^{*}-\left(\mathbf{F}_{2}^{T} / F_{44}\right) D_{2}^{\infty} .
$$

The solution to Eq. (34) is given by 


$$
\left\langle g_{i}\left(x_{1}\right)\right\rangle \mathbf{b}^{*}=\mathbf{F}_{1}^{*-1} \mathbf{T}^{*} \frac{x_{1}}{\left(a^{2}-x_{1}^{2}\right)^{1 / 2}} .
$$

Substituting Eq. (36) into Eqs. (33b) and (33c) yields

$$
\begin{aligned}
& \int_{-c}^{c} \frac{1}{\pi\left(x_{1}-x_{1}^{\prime}\right)} F_{44} g_{4} \Delta \varphi d x_{1}^{\prime}+D_{2}^{*}=0, \quad\left|x_{1}\right| \leq a, \\
& \int_{-c}^{c} \frac{1}{\pi\left(x_{1}-x_{1}^{\prime}\right)} F_{44} g_{4} \Delta \varphi d x_{1}^{\prime}+D_{2}^{*}=D_{b}^{*}, \quad a \leq\left|x_{1}\right| \leq c,
\end{aligned}
$$

where

$$
D_{2}^{*}=D_{2}^{\infty}-\mathbf{F}_{2} \mathbf{F}_{1}^{*-1} \mathbf{T}^{*}, \quad D_{b}^{*}=\left(E_{b}-E_{2}^{\infty}+\mathbf{G}_{1} \mathbf{F}_{1}^{*-1} \mathbf{T}^{*}\right) F_{44} / G_{2}+D_{2}^{*} .
$$

The solution to this singular integral equation of Eq. (37) is obtained by the method developed by Muskhelishvili (1953) and given by

$$
g_{4}\left(x_{1}\right) \Delta \varphi=\frac{D_{b}^{*}}{\pi F_{44}}\left[\operatorname{ch}^{-1}\left|\frac{c^{2}-a x_{1}}{c\left(a-x_{1}\right)}\right|-\operatorname{ch}^{-1}\left|\frac{c^{2}+a x_{1}}{c\left(a+x_{1}\right)}\right|\right] .
$$

The following equation must be satisfied to ensure the non-singularity at $\left|x_{1}\right|=c$

$$
\frac{a}{c}=\cos \left(\frac{\pi D_{2}^{*}}{2 D_{b}^{*}}\right)
$$

From Eq. (40) we can calculate the size of the dielectric breakdown zone,

$$
r_{b}=c-a=a \sec \left(\frac{\pi D_{2}^{*}}{2 D_{b}^{*}}\right)-a .
$$

Under the small yielding condition, $r_{b}<<a$, which corresponds to $\pi D_{2}^{*} /\left(2 D_{b}^{*}\right) \rightarrow 0$, Eq. (41) can be approximately reduced to

$$
r_{b}=\frac{a}{2}\left(\frac{\pi D_{2}^{*}}{2 D_{b}^{*}}\right)^{2}
$$

The stress ahead of the crack tip on the $x_{1}$-axis is calculated by 


$$
\begin{aligned}
& \Sigma_{2} \equiv\left(\begin{array}{llll}
\sigma_{12} & \sigma_{22} & \sigma_{32} & D_{2}
\end{array}\right)^{T} \\
& =\int_{-a}^{a} \frac{1}{\pi\left(x_{1}-x_{1}^{\prime}\right)}\left(\begin{array}{l}
\mathbf{F}_{1} \\
\mathbf{F}_{2}
\end{array}\right)\left\langle g_{i}\left(x_{1}^{\prime}\right)\right\rangle \mathbf{b}^{*} d x_{1}^{\prime}+\int_{-c}^{c} \frac{1}{\pi\left(x_{1}-x_{1}^{\prime}\right)}\left(\begin{array}{l}
\mathbf{F}_{2}^{T} \\
F_{44}
\end{array}\right) g_{4}\left(x_{1}^{\prime}\right) \Delta \varphi d x_{1}^{\prime}+\mathbf{t} .
\end{aligned}
$$

Using the definition of the intensity factor vector, $\mathbf{K}=\lim _{x_{1} \rightarrow a} \sqrt{2 \pi\left(x_{1}-a\right)} \Sigma_{2}$, we obtain

$$
\mathbf{K}^{(l)}=\sqrt{\pi a}\left(\begin{array}{l}
\mathbf{F}_{1} \\
\mathbf{F}_{2}
\end{array}\right) \mathbf{F}_{1}^{*-1} \mathbf{T}^{*},
$$

where the superscript " $(l)$ " on a parameter denotes the local value at the crack tip of the parameter.

Consequently, we have the local J-integral

$$
J^{(l)}=\mathbf{K}^{(l)^{T}} \frac{\mathbf{H}}{4} \mathbf{K}^{(l)} .
$$

These results from the DB model will be discussed in detail for two cases that the poling direction is perpendicular to the crack and the poling direction is parallel to the crack.

\section{Discussion}

\subsection{A crack perpendicular to the poling direction}

Fig. 3 shows a finite crack perpendicular to the poling direction. In this case, the constitutive relationship is explicitly expressed by 


$$
\left\{\begin{array}{l}
\sigma_{11} \\
\sigma_{22} \\
\sigma_{33} \\
\sigma_{32} \\
\sigma_{31} \\
\sigma_{12}
\end{array}\right\}=\left[\begin{array}{cccccc}
c_{11} & c_{13} & c_{12} & 0 & 0 & 0 \\
c_{13} & c_{33} & c_{13} & 0 & 0 & 0 \\
c_{12} & c_{13} & c_{11} & 0 & 0 & 0 \\
0 & 0 & 0 & c_{44} & 0 & 0 \\
0 & 0 & 0 & 0 & \left(c_{11}-c_{12}\right) / 2 & 0 \\
0 & 0 & 0 & 0 & 0 & c_{44}
\end{array}\right]\left\{\begin{array}{c}
\varepsilon_{11} \\
\varepsilon_{22} \\
\varepsilon_{33} \\
2 \varepsilon_{32} \\
2 \varepsilon_{31} \\
2 \varepsilon_{12}
\end{array}\right\}-\left[\begin{array}{ccc}
0 & e_{31} & 0 \\
0 & e_{33} & 0 \\
0 & e_{31} & 0 \\
0 & 0 & e_{15} \\
e_{15} & 0 & 0 \\
0 & 0 & 0
\end{array}\right]\left\{\begin{array}{l}
E_{1} \\
E_{2} \\
E_{3}
\end{array}\right\},
$$

$$
\left\{\begin{array}{l}
D_{1} \\
D_{2} \\
D_{3}
\end{array}\right\}=\left[\begin{array}{cccccc}
0 & 0 & 0 & 0 & e_{15} & 0 \\
e_{31} & e_{33} & e_{31} & 0 & 0 & 0 \\
0 & 0 & 0 & e_{15} & 0 & 0
\end{array}\right]\left\{\begin{array}{c}
\varepsilon_{11} \\
\varepsilon_{22} \\
\varepsilon_{33} \\
2 \varepsilon_{32} \\
2 \varepsilon_{31} \\
2 \varepsilon_{12}
\end{array}\right\}+\left[\begin{array}{ccc}
\kappa_{11} & 0 & 0 \\
0 & \kappa_{33} & 0 \\
0 & 0 & \kappa_{11}
\end{array}\right]\left\{\begin{array}{l}
E_{1} \\
E_{2} \\
E_{3}
\end{array}\right\} .
$$

For simplicity, remotely uniform loads considered here are $\sigma_{22}^{\infty}$ and $E_{2}^{\infty}$ and the other loading components are zero. Using the constitutive relationships, we have

$$
D_{2}^{\infty}=\hat{\kappa}_{\perp} E_{2}^{\infty}+\hat{e}_{\perp} \sigma_{22}^{\infty} \text { and } D_{1}^{\infty}=D_{3}^{\infty}=0
$$

where $\hat{\kappa}_{\perp}=\kappa_{33}+\mathbf{e}_{\perp}^{T} \mathbf{s}_{\perp} \mathbf{e}_{\perp}, \quad \mathbf{e}_{\perp}=\left(\begin{array}{lll}e_{31} & e_{33} & e_{31}\end{array}\right)^{T}, \quad \mathbf{s}_{\perp} \quad$ is the inverse matrix of $\mathbf{c}_{\perp}=\left(\begin{array}{lll}c_{11} & c_{13} & c_{12} \\ c_{13} & c_{33} & c_{13} \\ c_{12} & c_{13} & c_{11}\end{array}\right)$, and $\hat{e}_{\perp}=\mathbf{e}_{\perp}^{T} \mathbf{s}_{\perp}\left(\begin{array}{lll}0 & 1 & 0\end{array}\right)^{T}$. The $\mathbf{H}$ and $\mathbf{H}^{-1}$ matrixes take the forms (Wang, 2000):

$$
\mathbf{H}=\left(\begin{array}{cccc}
\frac{2}{C_{L}} & 0 & 0 & 0 \\
0 & \frac{2}{C_{T}} & 0 & \frac{2}{e} \\
0 & 0 & \frac{2}{C_{A}} & 0 \\
0 & \frac{2}{e} & 0 & -\frac{2}{\kappa}
\end{array}\right),
$$




$$
\mathbf{H}^{-1}=\left(\begin{array}{cccc}
\frac{C_{L}}{2} & 0 & 0 & 0 \\
0 & \frac{C_{T}}{2 \rho_{0}} & 0 & \frac{C_{T} \kappa}{2 \rho_{0} e} \\
0 & 0 & \frac{C_{A}}{2} & 0 \\
0 & \frac{C_{T} \kappa}{2 \rho_{0} e} & 0 & -\frac{\kappa}{2 \rho_{0}}
\end{array}\right) .
$$

where $\rho_{0}=1+C_{T} \kappa / e^{2}$. From Eqs. (31b), (35) and (48b), we have

$$
\begin{aligned}
\mathbf{F}_{1}^{*} & =\frac{1}{2}\left(\begin{array}{ccc}
C_{L} & 0 & 0 \\
0 & C_{T} & 0 \\
0 & 0 & C_{A}
\end{array}\right), \\
\mathbf{T}^{*} & =\left(\begin{array}{lll}
0 & \sigma_{22}^{\infty}+D_{2}^{\infty} C_{T} / e & 0
\end{array}\right)^{T} .
\end{aligned}
$$

Then, the local intensity factor vector is explicitly expressed by

$$
\mathbf{K}^{(l)}=\frac{\sqrt{\pi a}}{\rho_{0}}\left(\sigma_{22}^{\infty}+D_{2}^{\infty} C_{T} / e\right)\left(\begin{array}{c}
0 \\
1 \\
0 \\
\kappa / e
\end{array}\right) \text {. }
$$

Eq. (50) indicates that the local mode I stress intensity factor and the local intensity factor of electric displacement have nonzero values, which can be rewritten in terms of the applied ones:

$$
\begin{aligned}
& K_{I}^{(l)}=\frac{1}{\rho_{0}}\left(K_{I}^{(a)}+K_{D}^{(a)} C_{T} / e\right), \\
& K_{D}^{(l)}=\frac{\kappa}{e} K_{I}^{(l)}=\frac{\kappa}{\rho_{0} e}\left(K_{I}^{(a)}+K_{D}^{(a)} C_{T} / e\right) .
\end{aligned}
$$

Using Eqs. (22), (48a) and (51), we have the local energy release rate, which is given by

$$
J_{D B}^{(l)}=\frac{1}{2}\left(\frac{1}{C_{T}}+\frac{\kappa}{e^{2}}\right)\left(K_{I}^{(l)}\right)^{2}=\frac{1}{2 C_{T} \rho_{0}}\left(K_{I}^{(a)}+K_{D}^{(a)} \frac{C_{T}}{e}\right)^{2} .
$$


Wang (2000) derived the intensity factors from the PS model, with which we calculated the local energy release rate, $J_{P S}^{(l)}$, in terms of the applied stress intensity factor and the applied electric intensity factor:

$$
J_{P S}^{(l)}=\frac{1}{2 C_{T}}\left(K_{I}^{(a)}+K_{D}^{(a)} \frac{C_{T}}{e}\right)^{2} .
$$

Comparing Eq. (52) with Eq. (53), we find

$$
\frac{J_{P S}^{(l)}}{J_{D B}^{(l)}}=\rho_{0}=1+\frac{C_{T} \kappa}{e^{2}}>1 .
$$

This means that the PS model gives a higher value of the local energy release rate than that derived from the DB model and this phenomenon has been observed in the previous works (Zhang and Gao, 2004; Zhang, 2004). However, Eqs. (52) and (53) both indicate that positive electric field will assist an applied mechanical stress to propagate the impermeable crack if the local $\mathrm{J}$-integral is adopted as a failure criterion, while a negative electric field will retard crack propagation. Note that to ensure the crack open, the local mode I stress intensity factor must be positive, which requires $K_{I}^{(a)}>\left|K_{D}^{(a)}\right| C_{T} / e$ if the electrical field is negative. The relationship between the applied stress intensity factor and the applied electric intensity factor is identical in the two models. In this sense, the DB model gives the same result as the PS model.

As described above, the energy release rate can be apparently divided in the electric energy release rate and mechanical energy release rate. Due to the piezoelectric effect, an electric field contributes to the mechanical release rate and a stress field is involved in the electric energy release rate. In the DB model the electric field strength at the crack tip is completely shielded by the dielectric breakdown zone, whereas the electric displacement at the crick tip is completely shielded by the polarization saturation zone in the PS model. Either the complete shielding of the electric field strength or the complete shielding of the electric displacement leads to a zero 
value of the local electric energy release rate and results in the local energy release rate to be purely local mechanical energy release rate.

When the local energy release rate is purely mechanical, applying the local stress intensity factor as a failure will be equivalent to applying the local energy release rate. In the DB model, the local stress intensity factor is given by $K_{I, D B}^{(l)}=\frac{1}{\rho_{0}}\left(K_{I}^{(a)}+K_{D}^{(a)} C_{T} / e\right)$, whereas the local stress intensity factor is given by $K_{I, P S}^{(l)}=K_{I}^{(a)}+K_{D}^{(a)} C_{T} / e$ in the PS model (Wang, 2000). Clearly, the ratio of $K_{I, P S}^{(l)} / K_{I, D B}^{(l)}$ is the same as that of $J_{P S}^{(l)} / J_{D B}^{(l)}$, as shown in Eq. (54).

\subsection{A crack parallel to the poling direction}

In a similar manner, we describe the results for a finite crack parallel to the poling direction, which is shown in Fig. 4. In this case, the constitutive relationship is explicitly expressed by

$$
\left\{\begin{array}{l}
\sigma_{11} \\
\sigma_{22} \\
\sigma_{33} \\
\sigma_{32} \\
\sigma_{31} \\
\sigma_{12}
\end{array}\right\}=\left[\begin{array}{cccccc}
c_{33} & c_{13} & c_{13} & 0 & 0 & 0 \\
c_{13} & c_{11} & c_{12} & 0 & 0 & 0 \\
c_{13} & c_{12} & c_{11} & 0 & 0 & 0 \\
0 & 0 & 0 & \left(c_{11}-c_{12}\right) / 2 & 0 & 0 \\
0 & 0 & 0 & 0 & c_{44} & 0 \\
0 & 0 & 0 & 0 & 0 & c_{44}
\end{array}\right]\left\{\begin{array}{c}
\varepsilon_{11} \\
\varepsilon_{22} \\
\varepsilon_{33} \\
2 \varepsilon_{32} \\
2 \varepsilon_{31} \\
2 \varepsilon_{12}
\end{array}\right\}-\left[\begin{array}{ccc}
e_{33} & 0 & 0 \\
e_{31} & 0 & 0 \\
e_{31} & 0 & 0 \\
0 & 0 & 0 \\
0 & 0 & e_{15} \\
0 & e_{15} & 0
\end{array}\right]\left\{\begin{array}{l}
E_{1} \\
E_{2} \\
E_{3}
\end{array}\right\},
$$

$$
\left\{\begin{array}{l}
D_{1} \\
D_{2} \\
D_{3}
\end{array}\right\}=\left[\begin{array}{cccccc}
e_{33} & e_{31} & e_{31} & 0 & 0 & 0 \\
0 & 0 & 0 & 0 & 0 & e_{15} \\
0 & 0 & 0 & 0 & e_{15} & 0
\end{array}\right]\left\{\begin{array}{c}
\varepsilon_{11} \\
\varepsilon_{22} \\
\varepsilon_{33} \\
2 \varepsilon_{32} \\
2 \varepsilon_{31} \\
2 \varepsilon_{12}
\end{array}\right\}+\left[\begin{array}{ccc}
\kappa_{33} & 0 & 0 \\
0 & \kappa_{11} & 0 \\
0 & 0 & \kappa_{11}
\end{array}\right]\left\{\begin{array}{l}
E_{1} \\
E_{2} \\
E_{3}
\end{array}\right\}
$$


As did in the previous works (Gao et al., 1997; Wang, 2000), remotely uniform loads considered here are $\sigma_{22}^{\infty}$ and $E_{1}^{\infty}$ and the other loading components are zero. Using the constitutive relationships, we have

$$
D_{1}^{\infty}=\hat{\kappa}_{/ /} E_{1}^{\infty}+\hat{e}_{/ /} \sigma_{22}^{\infty} \text { and } D_{2}^{\infty}=D_{3}^{\infty}=0
$$

where $\quad \hat{\kappa}_{/ /}=\kappa_{33}+\mathbf{e}_{/ /}^{T} \mathbf{s}_{/ /} \mathbf{e}_{/ /}, \quad \mathbf{e}_{/ /}=\left(\begin{array}{lll}e_{33} & e_{31} & e_{31}\end{array}\right)^{T}, \quad \mathbf{s}_{/ /}$is the inverse matrix of $\mathbf{c}_{/ /}=\left(\begin{array}{lll}c_{33} & c_{13} & c_{13} \\ c_{13} & c_{11} & c_{12} \\ c_{13} & c_{12} & c_{11}\end{array}\right)$, and $\hat{e}_{/ /}=\mathbf{e}_{/ /}^{T} \mathbf{s}_{/ /}\left(\begin{array}{lll}0 & 1 & 0\end{array}\right)^{T}$. The $\mathbf{H}$ and $\mathbf{H}^{-1}$ matrixes take the forms (Wang, 2000):

$$
\begin{aligned}
\mathbf{H} & =\left(\begin{array}{cccc}
\frac{2}{C_{T}} & 0 & 0 & \frac{2}{e} \\
0 & \frac{2}{C_{L}} & 0 & 0 \\
0 & 0 & \frac{2}{C_{A}} & 0 \\
\frac{2}{e} & 0 & 0 & -\frac{2}{\kappa}
\end{array}\right), \\
\mathbf{H}^{-1} & =\left(\begin{array}{cccc}
\frac{C_{T}}{2 \rho_{0}} & 0 & 0 & \frac{C_{T} \kappa}{2 \rho_{0} e} \\
0 & \frac{C_{L}}{2} & 0 & 0 \\
0 & 0 & \frac{C_{A}}{2} & 0 \\
\frac{C_{T} \kappa}{2 \rho_{0} e} & 0 & 0 & -\frac{\kappa}{2 \rho_{0}}
\end{array}\right) .
\end{aligned}
$$

Then, from Eqs. (31b) and (35), we have

$$
\begin{gathered}
\mathbf{F}_{1}^{*}=\frac{1}{2}\left(\begin{array}{ccc}
C_{T} & 0 & 0 \\
0 & C_{L} & 0 \\
0 & 0 & C_{A}
\end{array}\right), \\
\mathbf{T}^{*}=\left(\begin{array}{lll}
0 & \sigma_{22}^{\infty} & 0
\end{array}\right)^{T} .
\end{gathered}
$$

Using Eq. (44), we express the local intensity factor vector explicitly as: 


$$
\mathbf{K}^{(l)}=\sqrt{\pi a}\left(\begin{array}{c}
0 \\
\sigma_{22}^{\infty} \\
0 \\
0
\end{array}\right) .
$$

Eq. (59) indicates that the only nonzero local intensity factor is the local mode I stress intensity factor, which is exactly the same as the applied one,

$$
K_{I}^{(l)}=K_{I}^{(a)} .
$$

Consequently the local energy release rate is given by

$$
J^{(l)}=\frac{2}{C_{L}}\left(K_{I}^{(l)}\right)^{2}=\frac{2}{C_{L}}\left(K_{I}^{(a)}\right)^{2}=J^{(a)} .
$$

Eqs. (60) and (61) indicate that the applied electric field does not change the mode I stress intensity factor and the energy release rate if the crack is parallel to the poling direction. The results from the DB model are consistent with the results from the PS model (Gao et al., 1997; Wang, 2000).

\section{Concluding remarks}

The present work reports the DB model for an electrically impermeable crack with a finite length based on the general linear constitutive equation. Although the general linear constitutive equation differs from the simplified constitutive equation used in the previous work (Zhang and Gao, 2004; Zhang, 2004), the derived results are qualitatively consistent with those reported in the previous work, as expected. In addition, comparing the obtained results from the PS model (Wang, 2000), it is found that the PS model gives a higher value of the local energy release rate than that derived from the DB model. However, both models are qualitatively consistent in the predication of the effect of electric fields on the fracture behavior. Finally, it should be noted that the analysis of the DB model has been restricted to the case of an impermeable crack to keep the consistency of the present work with the previous works (Gao et al., 1997; Wang, 2000; Zhang and Gao, 2004; Zhang, 2004), although the experimental results (Schneider et al., 2003) indicate 
that the permeable boundary condition might be super to the impermeable boundary condition in representing the real cases. We shall be able to theoretically extend the DB model to the cases of permeable cracks and/or semi-permeable cracks, however, the more challenging task is to experimentally verify the DB model.

Acknowledgements - This work was supported by a grant from the Research Grants Council of the Hong Kong Special Administrative Region, China. T.-Y Zhang thanks the Croucher Foundation for the Croucher Senior Research Fellowship Award, which gave him more research time by releasing him from teaching duties. M.-H. Zhao was supported by a distinguished young scholar science grant from Henan Province, China. 


\section{References}

Bilby, B. A., Cottrell, A. H. and Swinden, K. H. (1963). The spread of plastic yield from a notch. Proc. Roy. Soc. A 272, 304-314.

Cherepanov, G.P. (1979). Mechanics of Brittle Fracture. McGraw-Hill, New York.

Dissad, L. A. and Fothergill, J. C. (1992). Electrical degradation and breakdown in polymers. Peter Peregrinus Ltd., London.

Dugdale, D.S. (1960). Yielding of steel sheets containing slits. Journal of the Mechanics and Physics of Solids 8, 100-104

Gao, H. and Barnett, D. M. (1996). An invariance property of local energy release rates in a strip saturation model of piezoelectric fracture. International Journal of Fracture 79, R25-R29.

Gao, H.J., Zhang, T.Y. and Tong, P. (1997). Local and global energy release rates for an electrically yielded crack in a piezoelectric ceramic. Journal of the Mechanics and Physics of Solids 45, 491-510.

McMeeking, R.M. (2001). Towards a fracture mechanics for brittle piezoelectric and dieletric materials. International Journal of Fracture 108, 25-41.

Muskhelishvili, N.I. (1953). Singular Integral Equations. Noordhoff, Groningen.

$\mathrm{Ru}, \mathrm{C} . \mathrm{Q}$. (1999). Effect of electrical polarization on saturation on stress intensity factors in a piezoelectric ceramic. International Journal of Solids and Structures 36, 869-883.

Schneider, G.A., Felten, F. and McMeeking, R.M. (2003). The electrical potential difference across cracks in PZT measured by Kelvin probe microscopy and the implications for fracture. Acta Materialia 51, 2235-2241.

Wang, T.C. (2000). Analysis of strip electric saturation model of crack problem in piezoelectric materials. International Journal of Solids and Structures 37, 6031-6049.

Zhang, T.Y. and Tong, P. (1996). Fracture mechanics for a mode III crack in a piezoelectric material. International Journal of Solids and Structures 33, 343-359. 
Zhang, T.Y., Qian, C.F. and Tong, P. (1998). Linear Electro-elastic Analysis of a Cavity or a Crack in a Piezoelectric Material. International Journal of Solids and Structures 35, 21212149.

Zhang, T.Y., Zhao, M.H. and Tong, P. (2002). Fracture of piezoelectric ceramics. Advances in Applied Mechanics 38, 147-289.

Zhang, T.Y. and Gao, C.F. (2004). Fracture behaviors of piezoelectric materials. Theoretical and Applied Fracture Mechanics 41, 339-379.

Zhang, T.Y. (2004). Dielectric breakdown model for an electrical impermeable crack in a piezoelectric material. Computers, Materials \& Continua 1, 107-115. 


\section{$\underline{\text { List of Figure Captions }}$}

Fig. 1 An impermeable crack in a piezoelectric material under applied remote loadings.

Fig.2 The schematic distribution of an electric dislocation array in the DB model.

Fig.3 A crack perpendicular to the poling direction.

Fig.4 A crack parallel to the poling direction. 


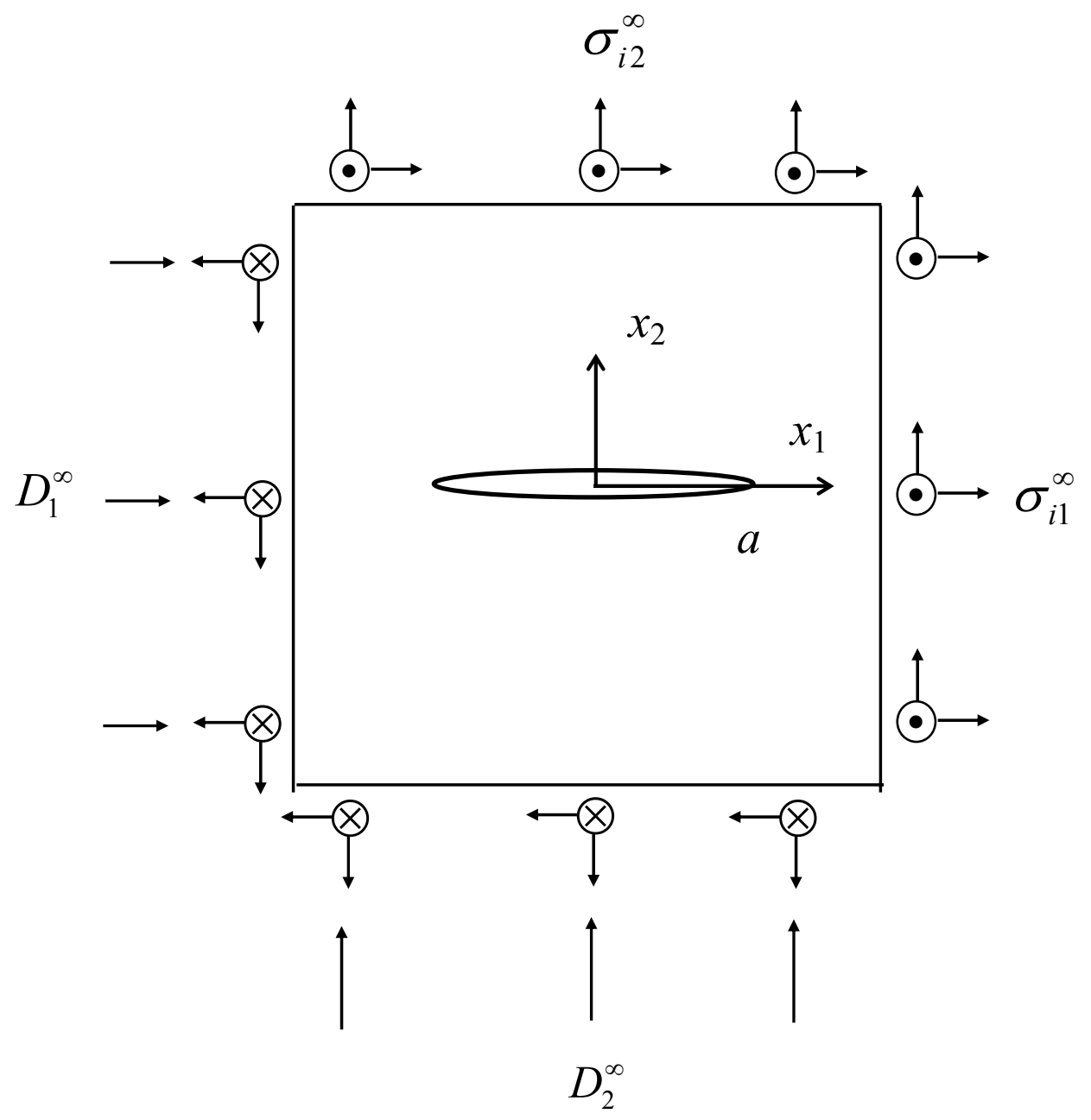

Fig. 1 An impermeable crack in a piezoelectric material under applied remote loadings. 


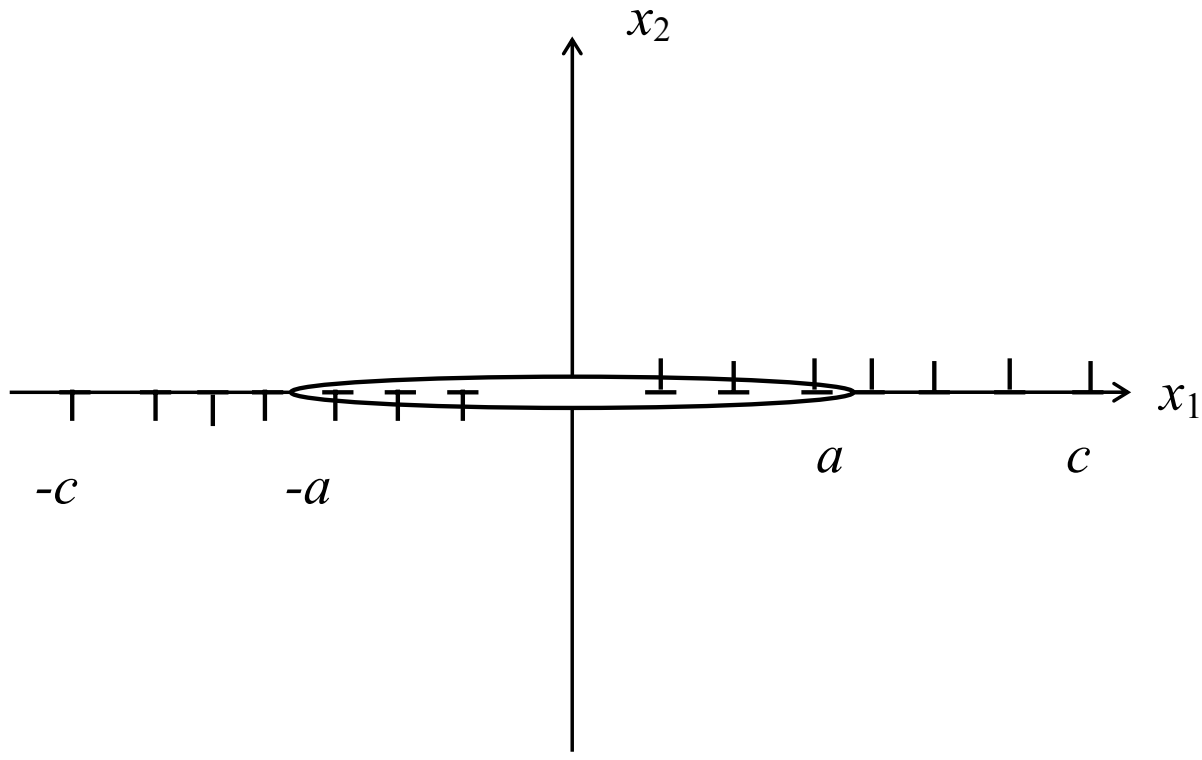

Fig.2 The schematic distribution of an electric dislocation array in the DB model. 


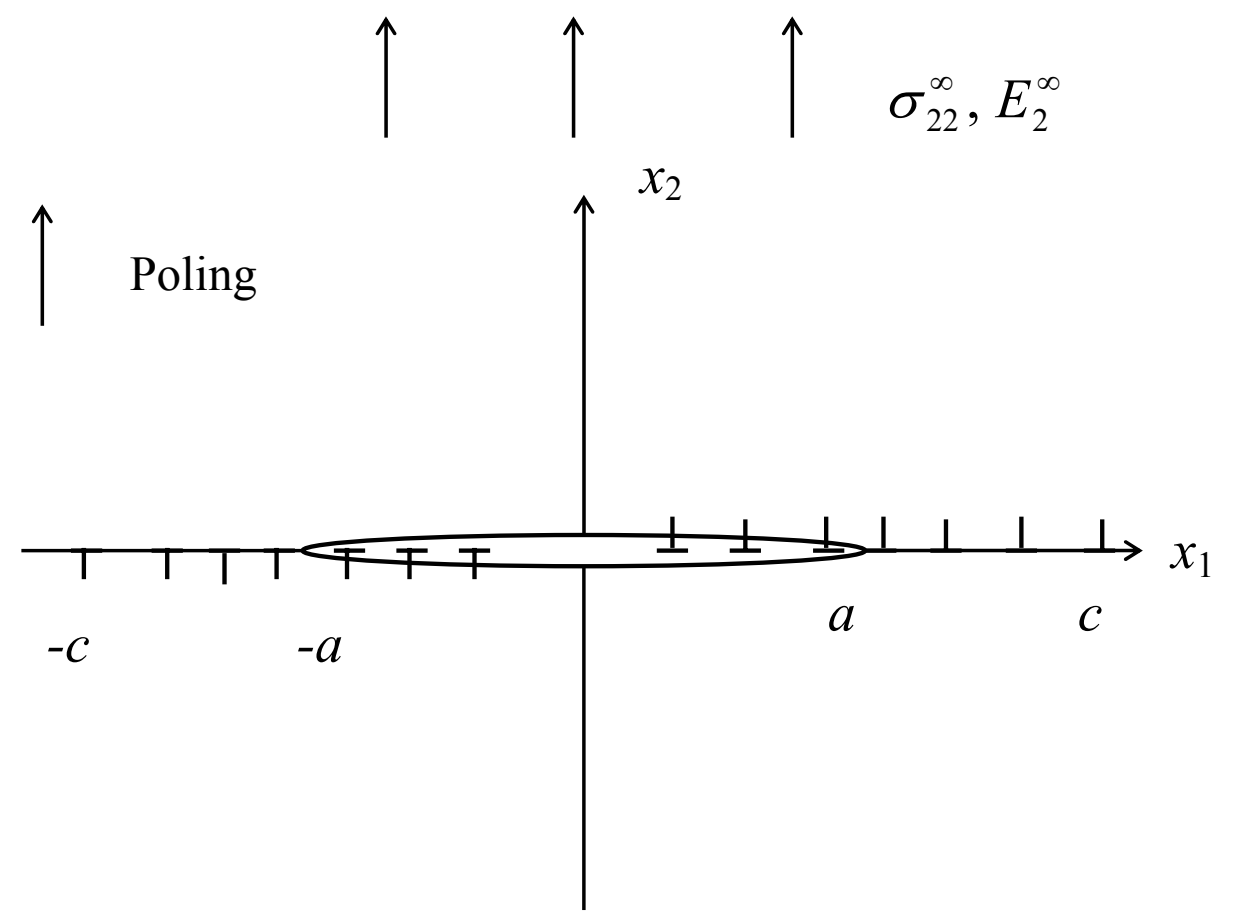

Fig.3 A crack perpendicular to the poling direction. 


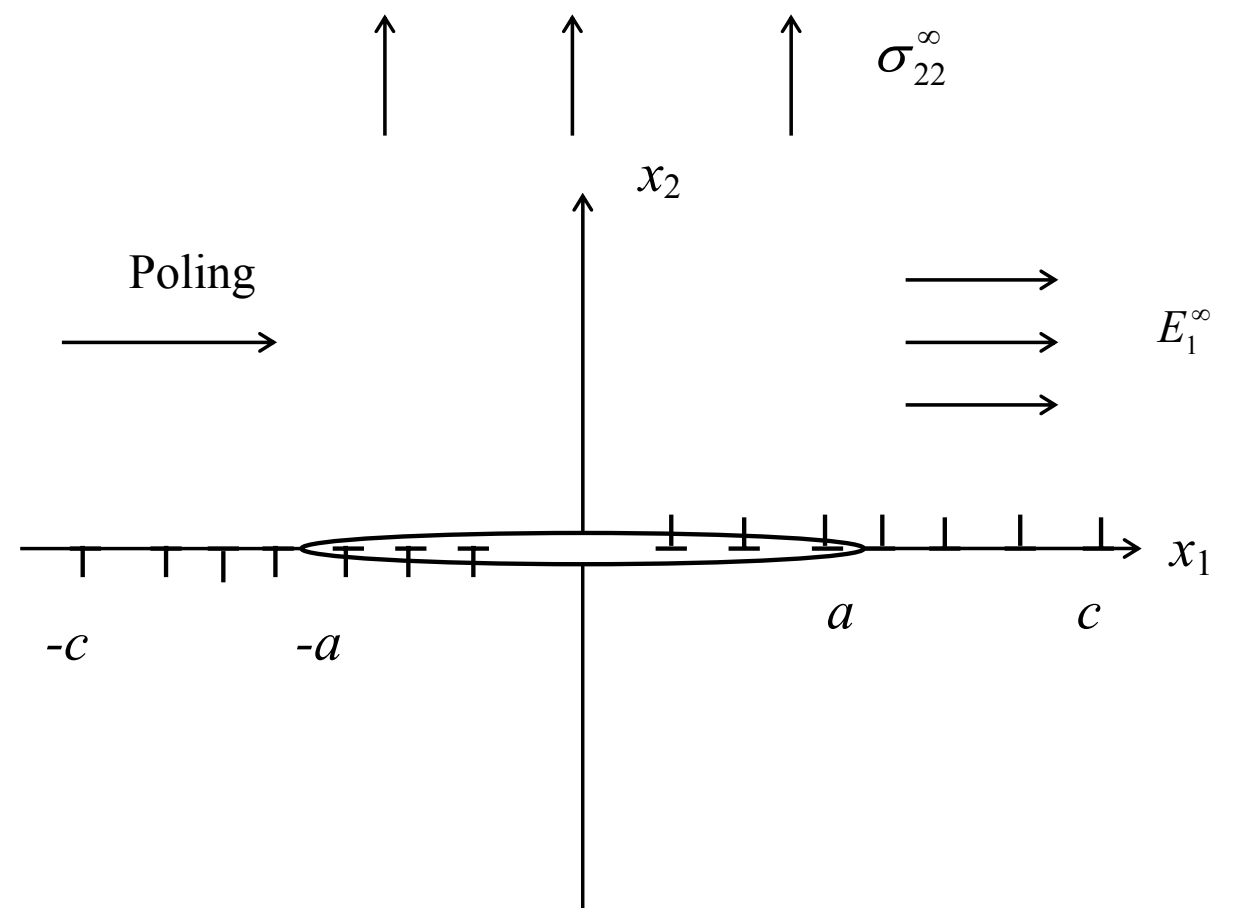

Fig.4 A crack parallel to the poling direction. 